\title{
Developmental characteristics of Tenebrio molitor larvae (Coleoptera: Tenebrionidae) in different instars
}

\author{
Jong Bin Park ${ }^{1}$, Won Ho $\mathrm{Choi}^{1}$, Seong Hyun $\mathrm{Kim}^{1}$, Hyo Jung Jin ${ }^{1}$, Yeon Soo $\mathrm{Han}^{2}$, Yong seok Lee ${ }^{3}$, \\ and Nam Jung $\mathrm{Kim}^{1 *}$ \\ ${ }^{1}$ Department of Agricultural Biology, National Academy of Agricultural Science, Suwon 441-100, Republic of Korea \\ ${ }^{2}$ Department of Plant Biotechnology, College of Agriculture \& Life, Chonnam National University, Kwangju 500-757, Republic of Korea \\ ${ }^{3}$ Department of Parasitology, College of Medicine, University of Soonchunhyang, Asan 336-745, Republic of Korea
}

\section{Abstract}

Tenebrio molitor is a major pest of stored grain, although its larvae have potential for use as food. However, little is known about the characteristics of individual larval instars of this species, and the number of instars remains disputed. Therefore, we assessed T. molitor for the average number of instars and its characteristics at different larval stages. The focus of this study was to establish a foundation for further studies on the characteristics of each larval instar. All of the T. molitor larvae showed incubation periods of 7 to $8 \mathrm{~d}$ and a period of 3 to $4 \mathrm{~d}$ for the $1^{\text {st }}$ instar. Beyond the $1^{\text {st }}$ instar, there were relatively large variations in the number of days in each instar period. Before emergence, most of the larvae had typically gone through 15 to 17 instars. The highest rate of pupae formation, $28.32 \%$, was observed in the $17^{\text {th }}$ instars. The body length gradually increased with each successive instar, reaching its maximum at the $17^{\text {th }}$ instar. Beyond the $17^{\text {th }}$ instar, however, the body length decreased. The larvae were white in the first instar, and gradually turned brown after the $2^{\text {nd }}$ instar.

(C) 2014 The Korean Society of Sericultural Sciences

Int. J. Indust. Entomol. 27(2), 5-9 (2014)

Received : 20 Dec 2013

Accepted : 20 Jan 2014

Keywords:

Tenebrio molitor,

larva,

body length,

instar

\section{Introduction}

Tenebrio molitor is a holometabolous insect that is considered to be a harmful pest of stored grain in South America (Schroeckenstein et al., 1990). However, its larvae, commonly referred to as mealworms, are used as pet food in many countries (Cotton, 1927). Because they are high in protein and fat and consume large amount of fiber, they represent a good food source for humans (Finke, 2002). Furthermore, they are high in oleic acid, which has the ability to decrease low-density lipoprotein (LDL) and increase high-density lipoprotein (HDL) levels in the blood (Yoo et al., 2013). In addition, they are inexpensive and easy to raise, with minimal harmful effects on the environment (Wang et al., 2012). For these reasons, many studies have been conducted on T. molitor to determine its potential uses.

The physiological features of $T$. molitor have been studied in some detail. Several studies on these aspects have found that the age of the parents influences the development of larvae; young parents are associated with the highest egg hatching rates (Ludwig and Fiore, 1960).

At the time of mating, the pheromone 4-methyl-1-nonanol is emitted by T. molitor females to attract males (Tanaka et al.,

\section{*Corresponding author.}

Nam Jung Kim

Applied Entomology Division, Department of Agricultural Biology, National Academy of Agricultural Science, RDA, Suwon 441-100, Republic of Korea

Tel: +82-31-290-8559 / FAX: +82-31-290-0109

E-mail: vastnj@korea.kr 
Table 1. Periods in each instar, number of pupae for each of the $14^{\text {th }}$ to $21^{\text {st }}$ instars, and body lengths of each larval stage in Tenebrio molitor.

\begin{tabular}{|c|c|c|c|c|}
\hline \multicolumn{2}{|c|}{ larval period } & \multicolumn{2}{|c|}{ number of pupae } & \multirow{2}{*}{$\frac{\text { body length }}{(\mathrm{cm})}$} \\
\hline instar & mean (days) & number & mean $(\%)$ & \\
\hline Incubation period & $7.47 \pm 0.74$ & - & - & - \\
\hline 1 & $3.39 \pm 0.51$ & - & - & $0.34 \pm 0.03$ \\
\hline 2 & $13.07 \pm 3.77$ & - & - & $0.39 \pm 0.01$ \\
\hline 3 & $10.24 \pm 3.11$ & - & - & $0.5 \pm 0.03$ \\
\hline 4 & $9.85 \pm 2.25$ & - & - & $0.64 \pm 0.03$ \\
\hline 5 & $10.71 \pm 2.19$ & - & - & $0.73 \pm 0.03$ \\
\hline 6 & $9.24 \pm 2.2$ & - & - & $0.8 \pm 0.03$ \\
\hline 7 & $8.66 \pm 2.09$ & - & - & $0.87 \pm 0.03$ \\
\hline 8 & $9.15 \pm 1.57$ & - & - & $0.96 \pm 0.09$ \\
\hline 9 & $9.16 \pm 1.57$ & - & - & $1.1 \pm 0.12$ \\
\hline 10 & $8.79 \pm 1.74$ & - & - & $1.34 \pm 0.19$ \\
\hline 11 & $9.02 \pm 1.86$ & - & - & $1.55 \pm 0.16$ \\
\hline 12 & $9.23 \pm 1.79$ & - & - & $1.88 \pm 0.18$ \\
\hline 13 & $10.86 \pm 2.26$ & - & - & $2.19 \pm 0.29$ \\
\hline 14 & $12 \pm 3.37$ & $2 \pm 1.73$ & $5.63 \pm 4.96$ & $2.63 \pm 0.22$ \\
\hline 15 & $11.78 \pm 3.25$ & $6.67 \pm 4.73$ & $19.39 \pm 15.94$ & $2.73 \pm 0.17$ \\
\hline 16 & $13.4 \pm 2.77$ & $8.33 \pm 4.73$ & $21.98 \pm 11.4$ & $2.94 \pm 0.11$ \\
\hline 17 & $14.71 \pm 4.79$ & $10.33 \pm 3.21$ & $28.32 \pm 8.42$ & $3.16 \pm 0.13$ \\
\hline 18 & $19.31 \pm 5.2$ & $5.33 \pm 2.52$ & $15.05 \pm 5.35$ & $2.61 \pm 0.71$ \\
\hline 19 & $30.33 \pm 8.08$ & $2.33 \pm 1.53$ & $6.29 \pm 3.68$ & $3.14 \pm 0.01$ \\
\hline 20 & $19 \pm 25.56$ & $0.67 \pm 1.73$ & $2.5 \pm 4.33$ & $3.16 \pm 0$ \\
\hline
\end{tabular}

The values are indicated as mean $\pm \mathrm{SD}$.

1986). This was confirmed by a study that indicated that males misperceived a glass rod that had been coated with 4-methyl1-nonanol as a female, and consequently displayed mating behaviors (Hurd and Parry, 1991).

The optimal temperature for the reproduction of T. molitor is $25-27.5^{\circ} \mathrm{C}$ as shown by Hein and Tracey (Fiore, 1960), while the total developmental time is 80.0-83.7 d (Park et al., 2012). In addition, they rapidly hide in grain when they are exposed to light, because they are characteristically nocturnal (Balfour and Carmichael, 1928). A decrease in humidity was found to be inconsequential to adults, larvae, or pupae at a temperature of $25^{\circ} \mathrm{C}$, but resulted in increased mortality at $10^{\circ} \mathrm{C}$ (Punzo and Mutchmor, 1980).
In the process of larval ecdysis, molting hormones play an important function to enable larvae to proceed to the next instar (Delbecque et al., 1978). Several studies have focused on changes in the number of instars in response to variation in oxygen density (Loudon, 1988), the change in the cuticle when T. molitor larvae molt into pupae and then adults, etc. (Delbecque et al., 1978). However, the characteristics of different larval stages remain unclear. In addition, the precise number of instars that $T$. molitor larvae go through at the optimal temperature of $25^{\circ} \mathrm{C}$ is debated. Therefore, this study was carried out to identify the characteristics of T. molitor larvae in different larval stages and to determine the average number of instars. The results of this study will help establish a foundation for investigating the 
various physiological characteristics of the different instars.

\section{Materials \& Methods}

\section{Breed condition}

T. molitor adults (about 1,000 ) were raised in acrylic boxes (width: $48 \mathrm{~cm}$, length: $49.5 \mathrm{~cm}$, height: $10.5 \mathrm{~cm}$ ) at $25^{\circ} \mathrm{C}$. Bran was laid on the bottom of each box, and a cabbage leaf was placed atop the bran layer as a source of moisture. Once a mating couple was observed, they were moved to a petri dish (diameter: $10 \mathrm{~cm}$, height: $1 \mathrm{~cm}$ ) containing bran. Eggs with hardened shells were obtained after 3-4 d. Subsequently, 45 eggs were collected. To measure the number of larval stages, each egg was transferred to a petri dish (diameter: $5 \mathrm{~cm}$, height: $1.5 \mathrm{~cm}$ ) containing bran and cabbage $(1 \mathrm{~g})$. This experiment was repeated 3 times.

\section{Measurement of body length of each instar}

After hatching, larval exuviae were counted to determine the number of instars and subsequently removed from the petri dishes. When the larva pupated, instar counting was stopped.

Another set of experiments was performed to measure the body length of each instar. The body lengths of 10 larvae were measured using Vernier Caliper each time an exuvium was observed. Generally, the width of the head capsule was measured because it exhibits distinct variation between larval stages (Hsia and Kao, 1987). In this experiment, however, the body lengths of larvae were measured owing to the small head capsule size during the early larval stages. Finally, photographs of each instar were taken using a DSLR camera.

\section{Results \& Discussion}

As shown in Table 1, the incubation period was 7-8 $\mathrm{d}$ and the duration of the $1^{\text {st }}$ instar was 3-4 d, on average. Interestingly, the larvae were not significantly different in terms of the incubation period or the duration of the $1^{\text {st }}$ instar. Between the $2^{\text {nd }}$ and $20^{\text {th }}$ instars, the duration of each instar was not uniform among larvae. Very few larvae were detected in the $19^{\text {th }}-20^{\text {th }}$ instar. The lack of uniformity among larvae between the $2^{\text {nd }}$ and 20 instars may be attributed to malnutrition. An experiment on Manduca sexta by Nijhout (1975) revealed that the number of instars increased when the larvae had a poor nutritional status. However, it remains to be verified if this phenomenon occurs in T. molitor. Furthermore, pathogens may also cause T. molitor larvae to exhibit symptoms of malnutrition. It was also revealed that a gregarious nature enhanced the ability of $T$. molitor to resist pathogens (Barnes and Siva-Jothy MT, 2000). Therefore, further studies need to be conducted to investigate whether the duration of each instar is influenced by poor nutritional status, pathogen activity, or larval behavior.

Pupation occurred after the $14^{\text {th }}$ instar. Approximately $69.69 \%$ of total pupation was observed between the $15^{\text {th }}$ and $17^{\text {th }}$ instars. The largest proportion of pupae (28.32\%) was observed in the $17^{\text {th }}$ instar. In other words, most of the larvae used in this experiment exhibited 15 to 17 instars in their life cycle. Before the $19^{\text {th }}$ instar, most of larvae pupated. Based on a study by Ludwig (1956), the average number of instars $25^{\circ} \mathrm{C}$ ranged from 11 to 15 (Fiore, 1960), which is smaller than what we found. This discrepancy may be explained by many factors, such as the nutritional status of the larvae and parents (Ludwig and Fiore, 1960). Repeating this study over a wider range of temperature may explain how ambient temperature influences the number of larval stages.

The body length of T. molitor instars increased gradually, reaching a maximum in the $17^{\text {th }}$ instar, and decreasing thereafter. This is because larvae with more than 17 instars were underdeveloped and therefore shorter than those in the $17^{\text {th }}$ instar. In addition, several pupae and dead larvae were detected between the $18^{\text {th }}$ and $20^{\text {th }}$ instars. Moreover, only 1 larva was observed in the $20^{\text {th }}$ instar. Owing to the massive occurrence of either pupation or mortality between the $17^{\text {th }}$ and $20^{\text {th }}$ instars, there was an insufficient number of larvae to accurately assess body length after the $17^{\text {th }}$ instar. Therefore, any study investigating this aspect must use a larger population of larvae.

Although the $1^{\text {st }}$ instar was white, the larvae gradually turned brown from and after the $2^{\text {nd }}$ instar. Except for the change in color, no significant differences in the morphological characteristics of the larvae were detected (Fig. 1)

Through this experiment, we confirmed the incubation period, the duration of the $1^{\text {st }}$ instar, and the average number of instars in T. molitor. These results may be useful as preliminary data for further studies on the characteristics of each instar period. 

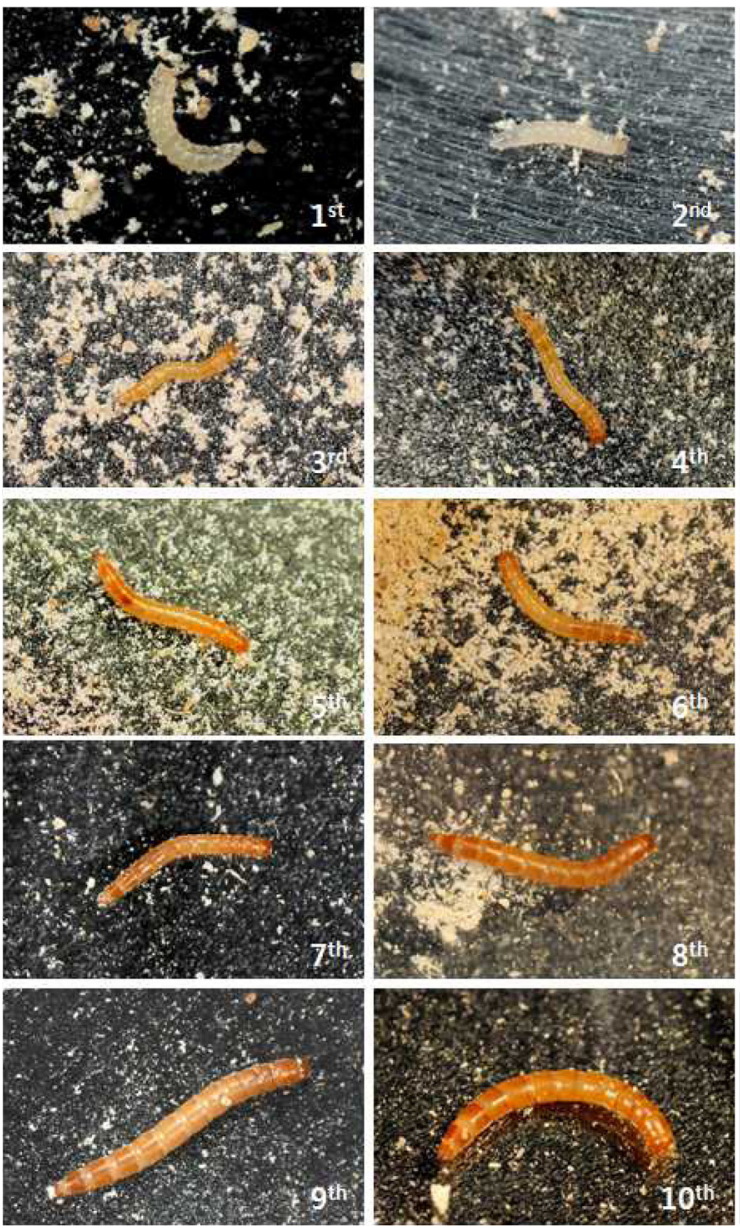
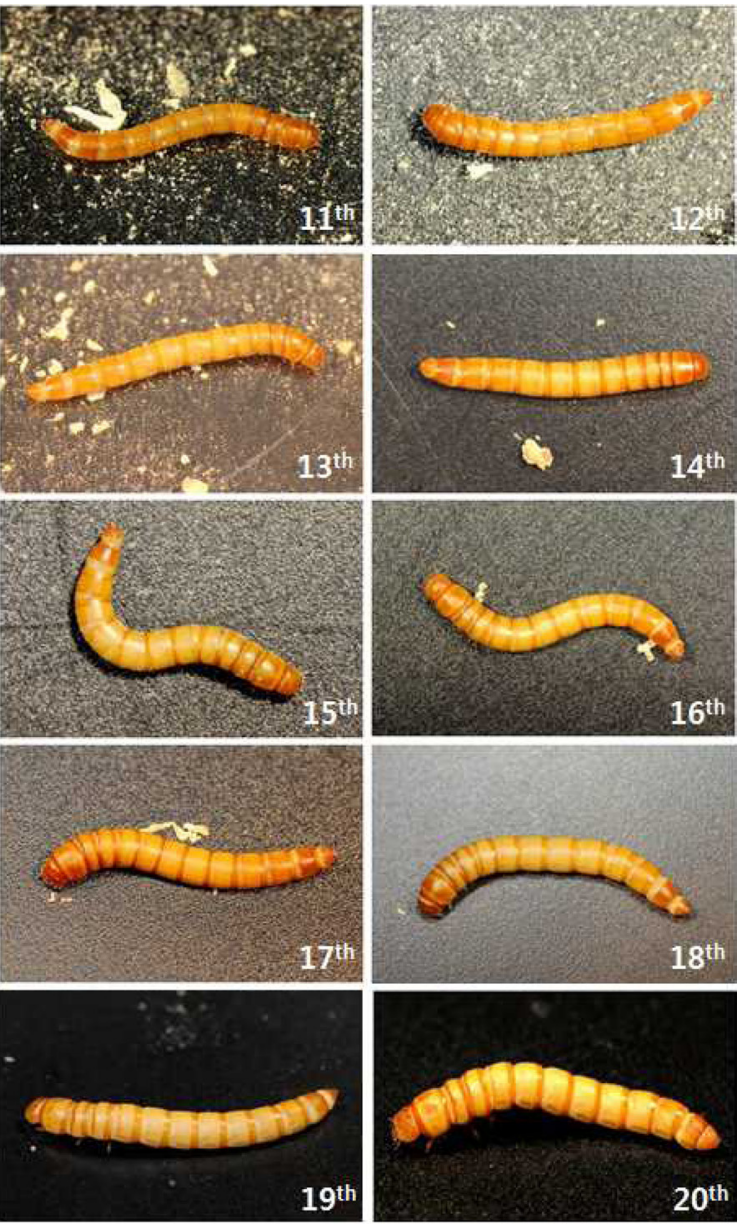

Fig. 1. Photographs of each larval instar. Photographs were taken using a DSLR camera after the exuvium was observed.

\section{Acknowledgments}

This study was carried out with support from the Research Program for Agricultural Science \& Technology Development (PJ 008186), National Academy of Agricultural Science, Rural Development Administration, and Republic of Korea.

\section{References}

Balfour CE, Carmichael L (1928) The light reactions of the mealworm (Tenebrio molitor Linn). Am J Psychol 40(4).

Barnes AI, Siva-Jothy MT (2000) Density-dependent prophylaxis in the mealworm beetle Tenebrio molitor L. (Coleoptera: Tenebrionidae): cuticular melanization is an indicator of investment in immunity. Proc R Soc Lond B 267(1439), 177-182.
Cotton RT (1927) Notes on the biology of the meal worms, Tenebrio molitor Linne and T. obscurus Fab. Ann Entomol Soc Am 20(1), 81-86(6).

Delbecque JP, Hirn M, Delachambre J, Reggi MD (1978) Cuticular cycle and molting hormone levels during the metamorphosis of Tenebrio molitor (Insecta Coleoptera). Developmental Biol 64, 11-30.

Finke MD (2002) Complete nutrient composition of commercially raised invertebrates used as food for insectivores. Zoo Biol 21, 269-285.

Fiore C (1960) Effects of temperature and parental age on the life cycle of the dark mealworm, Tenebrio obscurus Fabricius. J N Y Entomol Soc 68(1), 27-35.

Hsia WT, Kao SS (1987) Application of head width measurements for instar determination of corn earworm larvae. Taiwan R.O.C. 29, 277-282.

Hurd H, Parry G (1991) Metacestode-induced depression of 
the production of, and response to, sex pheromone in the intermediate host Tenebrio molitor. J Inverteb Pathol 58, 82-87.

Loudon C (1988) Development of Tenebrio molitor in low oxygen levels. J Insect Physiol 34, 97-103.

Ludwig D (1956) Effects of temperature and parental age on the life cycle of the mealworm, Tenebrio molitor Linnaeus (Coleoptera: Tenebrionidae). Ann Entomol Soc Am 49, 12-15.

Ludwig D, Carl F (1960) Further studies on the relationship between parental age and the life cycle of the mealworm, Tenebrio molitor. Ann Entomol Soc Am 53, 595-600(6).

Nijhout HF (1975) A threshold size for metamorphosis in the tobacco hornworm, Manduca Sexta (L.). Biol Bull 149, 214-225

Tanaka Y, Honda H, Ohsawa K, Yamamoto I (1986) A sex attractant of the yellow mealworm, Tenebrio molitor, and its role in mating behavior. J Pest Sci 11, 49-55.

Park YK, Choi YC, Lee SH, Lee JS, Kang SH (2012) Fecundity, life span, developmental periods and pupal weight of Tenebrio molitor L.(Coleoptera: Tenebrionidae). J Seric Entomol Sci 50(2), 126-132.

Punzo F and Mutchmor JA (1980) Effects of temperature, relative humidity and period of exposure on the survival capacity of Tenebrio molitor (Coleoptera: Tenebrionidae). J Kansas Entomol Soc 53(2), 260-270.

Schroeckenstein DC, Meier-Davis S, Bush RK (1990) Occupational sensitivity to Tenebrio molitor Linnaeus (yellow mealworm). J Allergy Clin Immunol 86(2), 182188.

Wang HC, Liao HY, Chen HL (2012) Tenebrio small-scale ecological farming feasibility study. Adv Mat Res, 267270.

Yoo JM, Hwang JS, Goo TW, Yun EY (2013) Comparative analysis of nutritional and harmful components in Korean and Chinese mealworms (Tenebrio molitor). J Korean Soc Food Sci Nutr 42(2), 249-254. 